\title{
Disfunção temporomandibular associada à cocleopatia: relato de caso
}

Temporomandibular dysfunction associated with cochleopathy: clinical case

Disfunción temporomandibular asociada con cocleopatía: reporte de caso Paula Gomes SOARES ${ }^{1}$

Lioney Nobre CABRAL ${ }^{2}$

${ }^{1}$ Graduanda em odontologia na Escola Superior de Ciências da Saúde - ESA/UEA, Universidade do Estado do Amazonas, 69.065-001 Manaus - AM, Brasil.

${ }^{2}$ Professor da Escola Superior de Ciências da Saúde - ESA/UEA, Universidade do Estado do Amazonas, 69.065-001 Manaus - AM, Brasil. Mestre em Patologia Tropical na Universidade Federal do Amazonas - UFAM, Manaus - AM, Brasil.

Doutor em Biotecnologia com concentração em Diagnóstico Bucal na Universidade Federal do Amazonas - UFAM, Manaus - AM, Brasil.

\section{Resumo}

Considerada uma deficiência funcional de ampla complexidade de sistematização dos sintomas e diagnósticos diferenciais, as disfunções temporomandibulares têm maior prevalência no gênero feminino, variando a faixa etária dos 18 aos 45 anos de idade. Pode estar associada a fatores dentários e/ou faciais, os quais se relacionam com o aparelho estomatognático. É imprescindível a anamnese criteriosa e avaliação física amiudada do sistema manducatório (sistema postural que integra músculos mastigatórios e cervicais) - campo multidisciplinar da odontologia, fonoaudiologia, otorrinolaringologia, neurologia e fisioterapia - para adequada propedêutica e assim, correto diagnóstico e precisa abordagem terapêutica do paciente. Este trabalho apresenta um relato de caso de paciente portadora de Disfunção Temporomandibular com alterações cocleares, diagnosticada, conduzida, tratada e evoluída em disciplina clínica de Estomatologia da Universidade do Estado do Amazonas. A problematização do caso, suas resoluções, e o compartilhamento dessas informações corroboram de modo considerável no aspecto clínico e científico, possibilitando ampliar o reconhecimento das diversas situações possíveis no atendimento aos pacientes com este quadro. Assim, as relações profissional-paciente e a multidisciplinaridade dos profissionais envolvidos ganham crescente relevância.

Descritores: Orelha Média; Sistema Estomatognático; Dor de Orelha; Cefaleia.

\section{Abstract}

Considered to be a functional deficiency of wide complexity of systematization of the symptoms and differential diagnoses, the temporomandibulares dysfunctions have a higher prevalence in the female gender, ranging from 18 to 45 years of age. It may be associated with dental and / or facial factors, which are related to the stomatognathic apparatus. Critical anamnesis and detailed physical evaluation of the manducatorio system (postural system that integrates masticatory and cervical muscles) are essential - multidisciplinary field of dentistry, phonoaudiology, otorhinolaryngology, neurology and physiotherapy - for proper propaedeutic and thus, correct diagnosis and accurate therapeutic approach of the patient. This paper presents a case report of a patient with Temporomandibular Dysfunction with cochlear alterations, diagnosed, conducted, treated and evolved in a clinical discipline of Stomatology of the State University of Amazonas. The problematization of the case, its resolutions and the sharing of this information corroborate in a considerable way in the clinical and scientific aspect, making possible to broaden the recognition of the various possible situations in the care of patients with this condition. Thus, the professional-patient relationship and the multidisciplinarity of the professionals involved gain increasing relevance.

Descriptors: Ear, Middle; Stomatognathic System; Earache; Headache.

\section{Resumen}

Considerada una deficiencia funcional de amplia complejidad de sistematización de los síntomas y diagnósticos diferenciales, las disfunciones temporomandibulares tienen mayor prevalencia en el género femenino, variando la franja etaria de los 18 a los 45 años de edad. Puede estar asociada a factores dentales y / o faciales, los cuales se relacionan con el aparato estomatognático. Es imprescindible la anamnesis criteriosa y evaluación física amiudada del sistema manducatorio (sistema postural que integra músculos masticatorios y cervicales) - campo multidisciplinario de la odontología, fonoaudiología, otorrinolaringología, neurología y fisioterapia - para adecuada propedéutica y así, correcto diagnóstico y preciso abordaje terapéutico del paciente. Este trabajo presenta un relato de caso de paciente portadora de Disfunción Temporomandibular con alteraciones cocleares, diagnosticada, conducida, tratada y evolucionada en disciplina clínica de Estomatología de la Universidad del Estado de Amazonas. La problemática del caso, sus resoluciones, y el compartir esas informaciones corroboran de modo considerable en el aspecto clínico y científico, posibilitando ampliar el reconocimiento de las diversas situaciones posibles en la atención a los pacientes con este cuadro. Así, las relaciones profesional-paciente y la multidisciplinariedad de los profesionales involucrados ganan creciente relevancia.

Descriptores: Oído Medio; Sistema Estomatognático; Dolor de Oído; Cefalea.

\section{INTRODUÇÃO}

A articulação temporomandibular (ATM), é uma estrutura altamente especializada do complexo craniomandibular que está sujeita a comprometimentos de origem neurológica, ortopédica e musculoesquelética, originando as disfunções temporomandibulares (DTMs). A função, saúde e estabilidade da ATM são essenciais para o desempenho harmonioso da mastigação, deglutição, fonação e postura ${ }^{1-3}$.

Desde os anos 30 do século XX a possibilidade da conexão entre disfunções temporomandibulares e tinnitus/otalgia tem sido discutida, levantando controvérsias. Variações do masseter e esternocleidomastóideo, músculos antigravitacionais da mastigação, podem alterar o padrão funcional do nervo vestibulococlear, responsável pelo movimento da cabeça, audição e equilíbrio, gerando sintomas otoneurológicos como vertigem, tontura e hipoacusia ${ }^{4}$.

Em 1934, James Costen, médico otorrinolaringologista americano, relatou pela primeira vez as desordens temporomandibulares, baseado em pesquisa com 11 pacientes, descrevendo suas condições oclusais e sintomas otoneurológicos ${ }^{5}$.

A origem comum da mandíbula e dos ossículos da orelha média, bem como a conexão cranial do ligamento timpanomandibular, capaz de mover o martelo durante a contração da cartilagem articular e ligamento esfenomandibular, explicaria a associação das DTMs com distúrbios auditivos. Outra hipótese seria a pressão produzida pelo deslocamento disto-posterior do côndilo mandibular sobre o nervo auriculotemporal e tuba auditiva ${ }^{6,7}$.

Estágios embriológicos explicam a íntima 
relação entre estruturas temporomandibulares e da orelha média. $\mathrm{O}$ primeiro arco branquial dará origem à mandíbula, maxila, arco zigomático e porção escamosa do osso temporal; o segundo arco branquial formará as regiões adjacentes do pescoço. O primeiro e segundo arco branquial formarão o pavilhão auditivo. Os músculos temporal, masseter, pterigóideo medial e lateral têm inserção ao redor das estruturas da orelha. Do músculo pterigóideo lateral provém o ligamento do martelo com a membrana timpânica. Os músculos palatinos têm vínculo anatômico com a boca e a tuba auditiva. Destarte fica elucidada a relação anatomofisiológica dessas estruturas $^{8,9}$.

Espasmos nos músculos mastigatórios induzem contração reflexa nos músculos tensor do tímpano e tensor do véu palatino. $O$ equilíbrio na tensão dos músculos do sistema locomotor da orelha média é de extrema importância para que todo o sistema não entre em disfunção e gere síndrome temporomandibular ${ }^{10}$.

Alterações fisiológicas, deslocamentos atípicos e forças oclusais excessivas podem gerar problemas funcionais da ATM com prejuízo às estruturas adjacentes, justificados pela proximidade anatômica da articulação temporomandibular com o meato acústico externo, bem como suas artérias, veias e nervos ${ }^{11}$. Dados da literatura demonstram relações morfológicas e funcionais dos ligamentos maleolares com a cápsula articular e estruturas mandibulares, que apesar de desempenharem tarefas distintas, mantém vínculo embriológico, anatômico e fisiológico ${ }^{12}$.

Para Costen e Louis ${ }^{5}$, a doença pode ser relacionada a problemas oclusais, onde a perda de dentes posteriores causa o deslocamento distal do côndilo, provocando pressão sobre estruturas retrocondilares. Também o estresse emocional é aceito como fator etiológico preponderante. No entanto, sabe-se que a etiologia é multifatorial, posto não haver causa isolada que justifique todos os sinais e sintomas. Deficiências nutricionais, distúrbios metabólicos, efeitos adversos de drogas, distúrbios esqueléticos, desgastes e perdas dentárias, restaurações e aparelhos protéticos insatisfatórios complementam a configuração etiológica ${ }^{5,13,14}$.

Os hábitos parafuncionais (mascar chiclete, roer unhas, apoio de mão na mandíbula, postura de mandíbula inadequada, fumar, morder objetos, sucção de bochechas/lábio, sucção digital ou de chupeta e bruxismo) são apontados como fatores de risco para a DTM e podem comprometer ossos, dentes e tecidos moles. As parafunções inibem o fluxo sanguíneo normal dos tecidos musculares, ocasionando acumulo de produtos metabólicos nas células destes tecidos, desencadeando sintomas de fadiga, dor e espasmo ${ }^{13,15,16}$.

O sintoma mais comum é a dor facial, aguda ou crônica, com flutuações de intensidade gerada pelo movimento mandibular. Surge diariamente ou com períodos livres de sintomas, podendo haver episódios de dor mais intensa associada ou não a sensação de plenitude auricular e hipoacusia devido a sensibilidade da musculatura mastigatória. A irradiação da dor para as regiões temporal, frontal, occipital, orbital, ângulo da mandíbula e meato acústico externo também é frequente ${ }^{17,18}$.

A percepção de sons, estalidos durante a fala ou alimentação e um ruído seco ou de choque rápido na superfície interna articular, que pode ser uni ou bilateral, é proveniente da deficiência de ligamentos articulares, falta de lubrificação da superfície articular pelo líquido sinovial ou, sendo potencialmente decorrente de degeneração na parte mais superficial das articulações ou desarranjo entre o côndilo e o disco ${ }^{15}$.

Também podem ocorrer a diminuição da dimensão vertical, limitação de abertura bucal, cefaleia, vertigem e tontura, odontalgia reflexa de origem miogênica, comprometimento do sinergismo muscular e desencadeamento de pontos-gatilhos nos locais de fixação dos músculos. Além de compressão de estruturas nervosas, que através de sinapses, levam informação ao sistema nervoso central, passando obrigatoriamente pelo $\mathrm{V}$ par do nervo craniano e, como resposta, surgem as dores referidas e até alterações autonômicas e disestésicas ${ }^{13,19}$.

O diagnóstico da cocleopatia a partir da alteração temporomandibular é de alta complexidade, por compreender muitos processos e uma vasta possibilidade propedêutica. Utiliza-se um sistema classificatório, aplicando um questionário, obtendo a queixa principal, a precisão da localização da dor, uma avaliação psicossocial do paciente e descobertas do exame físico. A avaliação otorrinolaringológica é imprescindível, pois objetiva excluir a possibilidade de quaisquer causas de origem primariamente otológica. Os estudos de imagem, em alguns casos, ajudam a esclarecer o diagnóstico. A radiografia panorâmica da mandíbula e a tomografia computadorizada auxiliam na detecção de mudanças degenerativas, presença de artropatias, neoplasias, alterações na posição e morfologia dos ossos, dentes e seios. Alguns procedimentos adicionais como as infiltrações de anestesia local com ou sem corticoide e as injeções de toxina botulínica podem contribuir nesse processo ${ }^{13,15,18}$.

Um estudo comparou pacientes portadores de DTM à pacientes com afecções otoneurológicas e apontou diferenças de dois grupos. Os sintomas prevalentes do grupo DTM foram otalgia e plenitude auricular, zumbido de aparecimento esporádico, como um apito de intensidade moderada e sem interferência nas atividades de vida diária. As queixas comuns do grupo de afecção otoneurológica foram hipoacusia e zumbido mais agudo, variando de 
intenso a muito intenso, sendo contínuo ou pulsátil, prejudicando as atividades de vida diária e o sono dos pacientes. Esses índices podem contribuir no diagnóstico, mas não como parâmetro único, pois doenças auditivas podem coexistir com a DTM, sem relação causa-efeito ${ }^{20}$.

São diagnósticos diferenciais da condição: subluxação mandibular, patologia dentária, doenças da glândula parótida, neuralgia trigeminal, glossofaríngea e/ou intermédia, câncer de cabeça, otites médias, disfunções da trompa de Eustáquio, artrite do osso temporal e cefaléia do tipo tensional ${ }^{18}$.

Muitos pacientes precisam de uma combinação de tratamento farmacológico e aconselhamento na posição apropriada ao dormir, reabilitações protéticas, uso de placas interoclusais miorrelaxantes, realização de exercícios fisioterápicos mandibulares e tratamento dos estados de ansiedade e depressão quando presentes. O tratamento inicial farmacológico é com associação de anti-inflamatório, analgésico, relaxante muscular e alcaloide (do tipo cafeína, para manter o estado de alerta, reduzindo o estado de sonolência provocado pelo relaxamento muscular), a cada 8 horas por 7 a 10 dias. Se a conduta não se tornar efetiva, utiliza-se antidepressivo tricíclico durante 4 meses. Os casos refratários ao tratamento conservador devem ser reavaliados. Assim, condutas como infiltrações com anestésico e/ou corticoide intra ou extra-articular, artrocentese, artroscopia, artrotomia aberta ou reconstrução mandibular podem ser consideradas ${ }^{18,21}$.

Os pacientes com DTM miogênica de menor complexidade podem ser tratados por orientações para autocuidado, exercícios e splints. Os casos mais complexos devem ser tratados por uma equipe multidisciplinar $^{22}$. A fisioterapia e a acupuntura são terapias de suporte que visam reduzir ou eliminar sinais e sintomas, mantendo ou recuperando a atividade funcional num menor espaço de tempo ${ }^{23,24}$.

O presente estudo teve como objetivo apresentar um caso de tratamento de uma paciente acometida por disfunção temporomandibular associada à cocleopatia.

\section{CASO CLÍNICO}

Paciente gênero feminino, leucoderma, 50 anos, brasileira, procedente da cidade de Manaus/AM, compareceu à Policlínica Odontológica da Universidade do Estado do Amazonas - UEA, Clínica de Estomatologia, com queixa de dores faciais intensas, mais frequentes do lado esquerdo. A paciente relatou sintomatologia dolorosa na região massetérica e pré-auricular, sensibilidade na ATM esquerda, presença de zumbido intenso e sensação de plenitude auricular na orelha esquerda. Relatou ainda cefaleias periódicas. Negou vertigens, alergias, traumas anteriores ou quaisquer alterações sistêmicas dignas de nota. Não houve relato com relação a hábitos parafuncionais. Ao exame clínico, observouse que a paciente apresentava assimetria facial devido ao aumento de volume do lado esquerdo da face e hábitos posturais nocivos (Figura 1).

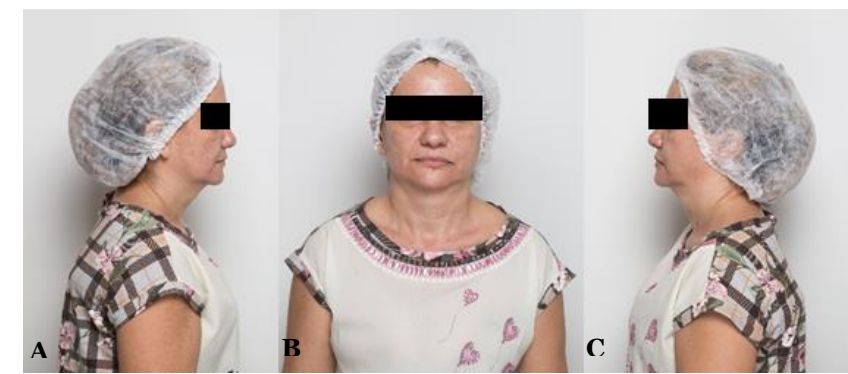

Figura 1: A. Registro do perfil direito; B. Registro frontal; C. Registro do perfil esquerdo.

Não observada presença de linfadenopatia, foi descartada a hipótese de dor de origem dentária. Com o auxílio de um paquímetro, foi possível mensurar a abertura máxima da boca (distância interincisal) em $40 \mathrm{~mm}$; restrição mandibular excêntrica em $4,5 \mathrm{~mm}$ do lado direito e $5 \mathrm{~mm}$ do lado esquerdo (Figura 2); interferência mandibular com deflexão na abertura para esquerda em $30 \mathrm{~mm}$, sem retorno à linha média; desvio em protrusão para esquerda.

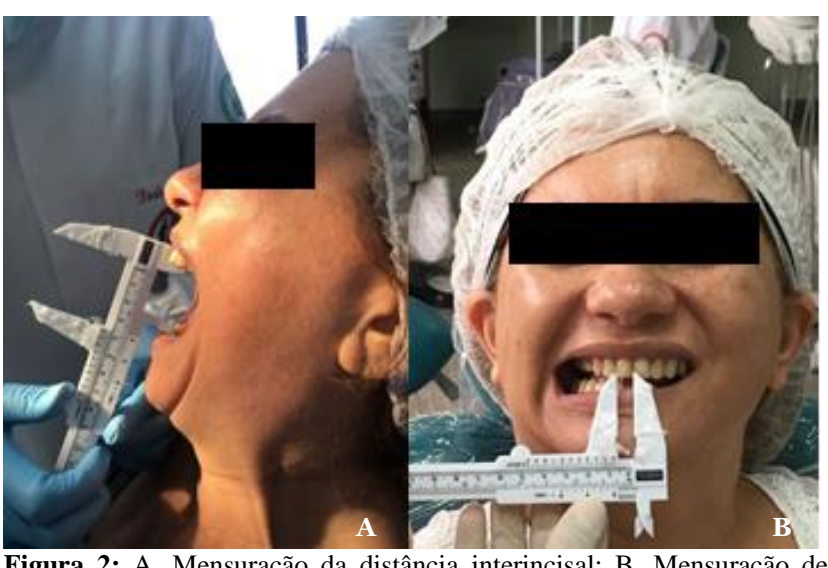

Figura 2: A. Mensuração da distância interincisal; B. Mensuração de restrição mandibular excêntrica.

Por meio da palpação bilateral dos músculos temporal (feixes anterior, médio e posterior), masseter (proximal, corpo e distal), esternocleidomastóideo (porções mastóidea, clavicular e esternal) e cervicais posteriores (Figura 3) notou-se a presença de pontos-gatilhos com diferentes graus de percepção dolorosa.

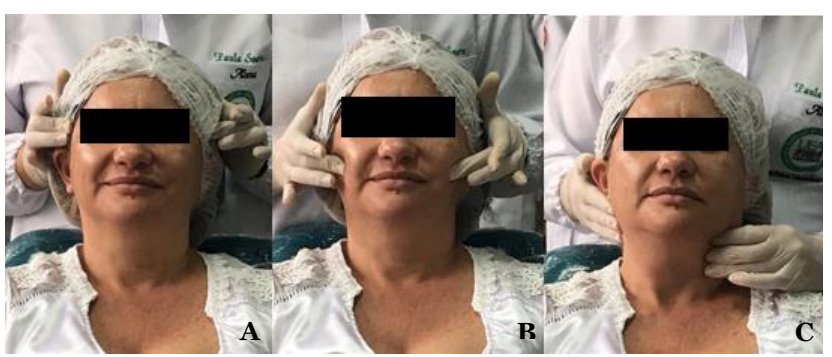

Figura 3: A. Palpação do músculo temporal; B. Palpação do músculo masseter; C. Palpação do músculo esternocleidomastoideo.

O limiar de dor foi registrado seguindo uma escala de 0 a 3, onde: (0) Paciente não relata 
desconforto quando o músculo é palpado; (1) Paciente relata desconforto ou dolorimento durante a palpação; (2) Paciente sente definitivamente desconforto ou dor; e (3) Paciente tem reação de fuga à palpação, lacrimeja, ou verbaliza o desejo de não querer que determinada área seja palpada novamente (Figura 4).

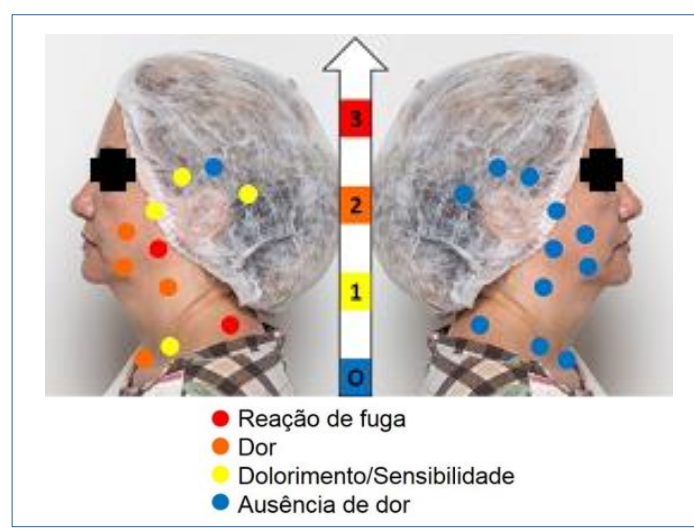

Figura 4: Avaliação inicial dos pontos-gatilhos e limiar de percepção dolorosa à palpação.

A propedêutica, após anamnese e exame físico geral e específico, conduziu à hipótese diagnóstica de Disfunção temporomandibular de origem muscular levando ao comprometimento coclear. $\mathrm{O}$ tratamento inicial proposto foi orientação da correção postural, indicação de fisioterapia da musculatura envolvida com exercícios mandibulares de abertura mínima de boca contra resistência (repetição de 10 vezes com duração de 10 segundos cada), fechamento de boca contra resistência (repetição de 4 vezes com duração de 10 segundos cada) e abertura e fechamento orientados pelos dedos indicador e polegar (repetição de 30 vezes com frequência de três vezes ao dia) (Figura 5), associada à termoterapia de compressa fria intermitente $\mathrm{e}$ concomitante aos exercícios em dias quentes e morna úmida sem intermitência e também concomitante, em dias frios.

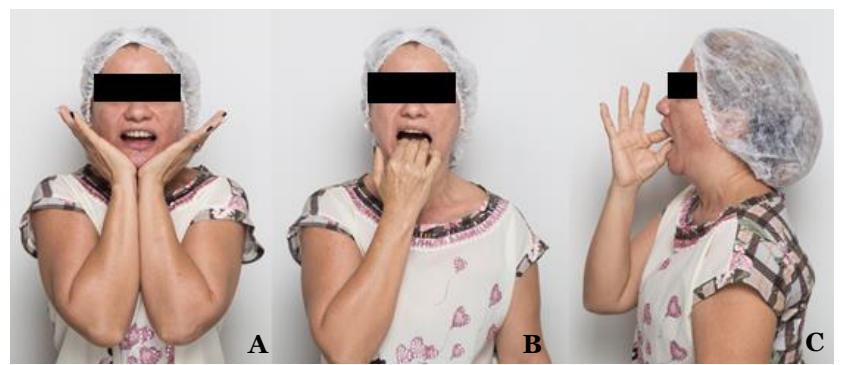

Figura 5: Paciente executando exercícios de: A. Abertura contra a resistência; B. Fechamento contra resistência; C. Abertura e fechamento orientados.

A terapia medicamentosa preconizada foi um composto sob a forma de comprimido associado de Diclofenaco de potássio (anti-inflamatório) $50 \mathrm{mg}$, paracetamol (analgésico) $300 \mathrm{mg}$, carisoprodol (relaxante muscular periférico) $125 \mathrm{mg}$ e um alcaloide estimulante central (cafeína) $30 \mathrm{mg}$, com o nome comercial de Tandrilax, tomado a cada 8 horas por 7 dias e um protetor gástrico (Pantoprazol $20 \mathrm{mg}$ ) antes do café da manhã durante o tempo de uso da medicação, pelo fato de ocorrer redução de prostaglandinas protetoras do estômago pela ação do anti-inflamatório. A segunda opção utilizada ocorreu para suporte da paciente, após um evento estressor na vigência do tratamento, sendo prescrito um relaxante muscular de ação central, Cloridrato de ciclobenzaprina (Miosan $10 \mathrm{mg}$ ), para ingesta às 22 horas, por 7 dias, com objetivo de tirar a paciente do estado agudo decorrente da reatividade deste tecido específico ao estresse, promovendo seu relaxamento. Em seguida, a paciente foi moldada para registro do modelo de estudo em gesso, montado em articulador semiajustável, a fim de realizar análise oclusal e articular e confecção de placa interoclusal (miorelaxante) em resina acrílica (Figura 6).

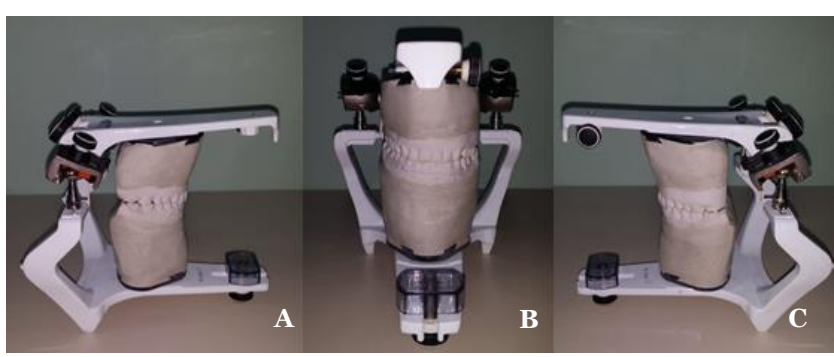

Figura 6: Modelo de estudo montado em articulador semi-ajustável em visão: A. Lateral direita; B. Frontal; C. Lateral esquerda.

A placa foi instalada e a paciente foi orientada ao uso contínuo, exceto durante as refeições (Figura 7). Em uma semana, o tratamento se revelou positivo com a redução dos sinais e sintomas. Foi observado um ganho na abertura mandibular, diminuição das restrições mandibulares e menor frequência de zumbidos na orelha esquerda. Durante o acompanhamento, nas consultas subsequentes, a paciente relatou que a sintomatologia reaparecia com oscilações de humor.

Após 18 meses de tratamento, a paciente apresentou melhora significativa do quadro clínico, onde a cefaleia estava ausente, o zumbido inexistente e a plenitude auricular era rara, houve um ganho de $5 \mathrm{~mm}$ na distância interincisal. Restrição mandibular direita ausente (inicial $4,5 \mathrm{~mm}$ e final $10 \mathrm{~mm}$ ) e restrição mandibular esquerda com diferença de $2 \mathrm{~mm}$ (inicial $5 \mathrm{~mm}$ e final $7 \mathrm{~mm}$ ).

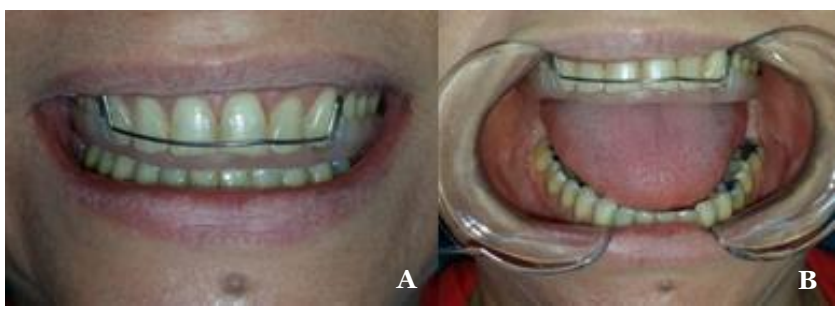

Figura 7: Instalação da placa interoclusal miorrelaxante. A. Registro em oclusão; B. Registro com a boca aberta.

Ao final do tratamento a paciente relatou sentir-se muito melhor com sintomatologia dolorosa quase inexistente (Figura 8). 
efetiva para o alívio da sintomatologia apresentada pela paciente.

\section{REFERÊNCIAS}

1. Miraglia SS, Nogueira RP, Monazzi MS, Soares FAV. Principais Problemas da ATM. Guia prático de orientação diagnóstica para o clínico geral. Rev Bras Prot Clin Lab. 2001;3(14):271-77.

2. Matta MAP, Honorato DC. Uma abordagem fisioterapêutica nas desordens temporomandibulares: estudo retrospectivo. Fisioter Pesq. 2003;10(2):77-83.

Figura 8: Avaliação final dos pontos - gatilhos e limiar de percepção dolorosa à palpação.

DISCUSSÃO

Ossos e músculos da orelha média são interpretados como instrumentos mastigatórios com função auditiva tendo em vista que existe uma fonte comum de inervação, proveniente do nervo mandibular e conexão funcional entre o aparelho mastigatório e órgão acústico ${ }^{11}$.

Oscilações do estado emocional do indivíduo podem causar aumento do tônus muscular e elevação da atividade gama eferente. As experiências passadas armazenadas no córtex e sistema límbico, em especial às relacionadas ao contexto afetivo e emocional, assim como o estado atual destas emoções, influenciam na elevação da percepção dolorosa. Neste caso clínico, quando a paciente era submetida a altos níveis de estresse e ansiedade, os sintomas como mialgia, fadiga muscular e restrição mandibular ficavam mais evidentes. Pacientes portadores de mialgia possuem seis vezes mais chances de apresentarem limitação de abertura bucal $^{13,25,26}$.

$\mathrm{O}$ tratamento foi estabelecido seguindo a avaliação da musculatura mastigatória e cervical, das dores presentes, da condição psicológica da paciente e da oclusão (interferências, parafunções, dimensão vertical) analisadas com o auxílio de modelos em gesso montados em articulador semiajustável que oferece a possibilidade de analisar tridimensionalmente os movimentos mandibulares planejados $^{27}$.

A instalação do dispositivo interoclusal auxiliou na redução da hiperatividade muscular, e estabilização da mandíbula, descomprimindo a articulação temporomandibular e devolvendo a dimensão vertical de oclusão à paciente, promovendo melhora nas condições da fala e da mastigação.

\section{CONSIDERAÇÕES FINAIS}

Os hábitos posturais corretos adotados pela paciente, o controle da ansiedade e os exercícios terapêuticos mostraram-se benéficos na melhora da condição dolorosa muscular, dos sintomas auriculares, dos movimentos mandibulares e no fortalecimento musculoesquelético, proporcionando sonos mais regulares ${ }^{23}$. Levando em consideração esses aspectos, a terapêutica aplicada mostrou-se

3. Pereira KNF, Andrade LLS, Costa ML, Portal TF. Sinais e sintomas de pacientes com disfunção temporomandibular. Rev CEFAC. 2005;7(2):221-28.

4. Seedorf H, Jude HD. Otalgia as a result of certain temporomandibular joint disorders. Laryngorhinootologie. 2006;85(5):327-32.

5. Costen JB, Louis ST. A syndrome of ear and sinus symptoms dependent upon disturbed function of thetemporomandibular joint 1934. Ann Otol Rhinol Laryngol. 1997;106(10 Pt 1):805-19.

6. Mota LAA, Albuquerque KMG, Santos MHP, Travassos RO. Sinais e sintomas associados à otalgia na disfunção temporomandibular. Arq Int Otorrinolaringol. 2007;11(4):411-15.

7. Pascoal MIN, Abrão R, Chagas JFS, Pascoal MPBN, Claudiney CC, Magna LA. Prevalência dos sintomas otológicos na desordem temperomandibular: estudo de 126 casos. Rev Bras Otorrinolaringol. 2001;67(5):627-33.

8. Sicher H. Temporomandibular articulation in mandibular overclosure. J Am Dent Assoc. 1948; 36(2):131-39.

9. Gardner E, Gray DJ, O'Rahilly R, Benevento RH. Anatomia: estudo regional do corpo humano. Rio de Janeiro: Guanabara Koogan; 1978.

10. Myrhaug $\mathrm{H}$. The incidence of ear symptoms in cases of malocclusion and temporomandibular joint disturbancez. Br J Oral Surg. 1964; 2(1):28-32.

11.Zocoli R, Mota ME, Sommavilla A, Perin LR. Manifestações otológicas nos distúrbios da articulação temporomandibular. ACM Arq Catarin Med. 2007;36(1):90-95.

12. Ramírez, LM, Ballesteros ALE, Sandoval OGP. A direct anatomical study of the morphology and functionality of disco-malleolar and anterior malleolar ligaments.Int J Morphol. 2009; 27(2):367-79.

13. Okeson PJ. Tratamento das desordens temporomandibulares e oclusão. São Paulo: Artes Médicas; 2000.

14. Camparis CM, Formigoni G, Teixeira MJ, de Siqueira JT. Clinical evaluation of tinnitus in patients with sleep bruxism: prevalence and characteristics. J Oral Rehabil. 2005;32(11):808-14. 
CONFLITO DE INTERESSES

Os autores declaram não haver conflitos de interesse. Medeiro ME, Gondim NFR, Barbosa RAD. Oclusão: noções e conceitos básicos. São Paulo: Santos; 1997.

16. Quinto AC. Classificação e Tratamento das Disfunções Temporomandibulares. Qual o papel do fonoaudiólogo no tratamento dessas disfunções? Rev CEFAC. 2000;2(2):15-22.

17. López-Zuazo A, Sánchez PM.A. Salinas Cubillas Servicio de Neurología. Hospital Universitario de Guadalajara. Guadalajara. España. Medicine. 2015;11:4184-97.

18. Scrivani SJ, Keith DA, Kaban LB. Temporomandibular disorders. N Engl J Med. 2008;359(25):2693-705.

19. Barreto DC, Barbosa ARC, Frizzo ACF. Relação entre disfunção temporomandibular e alterações auditivas. Rev CECAF. 2010;12(6):1067-76.

20. Felício CM, Oliveira JAA, Nunes LJ, Jeronymo LFG, Ferreira-Jeronymo RR. Alterações auditivas relacionadas ao zumbido nos distúrbios otológicos e da articulação temporomandibular. Rev Bras Otorrinolaringol. 1999;65(2):141-46.

21. Jorge JH, Silva Junior GS, Urban VM, Neppelenbroek KH, Bombarda NHC. Desordens temporomandibulares em usuários de prótese parcial removível: prevalência de acordo com a classificação de Kennedy. Rev Odontol UNESP. 2013;42(2):72-7.

22. Fricton J. Myogenous temporomandibular disorders: diagnostic and management considerations. Dent Clin North Am. 2007; 51(1):61-83.

23. Feine JS, Widmer CG, Lund JP. Physical therapy: a critique. Oral Surg Oral Med Oral Pathol Oral Radiol Endod. 1997;83(1):123-27.

24. Alves Rezende MCR, Marques CB, Gonçales AN, Sales A, Ávila SMHC, Magalhães AP et al. Sinais e sintomas na síndrome de Costen associada a desordens temporomandibulares: relato de caso clínico. Revista Odontol Araçatuba. 2011;32(1):65-9.

25. Garcia AR. Desordens Temporomandibulares. In: Madeira MC. Anatomia da face. São Paulo: Sarvier; 2010.

26. Figueiredo VMG, Cavalcanti AL, Farias ABL e Nascimento SR. Prevalência de sinais, sintomas e fatores associados em portadores de disfunção temporomandibular. Acta Sci Health Sci. 2009; 31(2):159-63.

27. Ash CM, Pinto OF. The tmj and the mddle ear: structural and functional correlates for aural symptoms associated with temporomandibular joint dysfunction. Int $\mathrm{J}$ Prosthodont. 1991;4(1):51-7.
Submetido em 19/03/2019

Aceito em 11/06/2019

\section{AUTOR PARA CORRESPONDENCIA}

\section{Paula Gomes Soares}

marquespgs@gmail.com 